EPJ Web of Conferences 59, 05017 (2013)

DOI: $10.1051 /$ epjconf/20135905017

(C) Owned by the authors, published by EDP Sciences, 2013

\title{
Modelling of ion deceleration in interpenetrating laser-generated plasma plumes
}

\author{
O. Larroche ${ }^{1}$, R. Liska ${ }^{2}$, O. Renner ${ }^{3}$ and E. Dalimier ${ }^{4}$ \\ 1 CEA DIF, Bruyères le Châtel, Arpajon, France \\ 2 Czech Technical University in Prague, FNSPE, Prague, Czech Republic \\ 3 Institute of Physics, v.v.i., Czech Academy of Sciences, Prague, Czech Republic \\ 4 Université Pierre et Marie Curie UPMC, LULI, Paris, France
}

\begin{abstract}
The modelling of intense laser interaction with solid targets represents a complicated complex of plasma physics and numerical methods. The presented simulations contribute to a better understanding of plasma-wall interactions by studying the interpenetration and mutual deceleration of two plasma plumes of different materials created on two foil targets. The initial phase is simulated by the $2 \mathrm{D}$ hydrodynamics code PALE until the plumes start to interpenetrate. The mixing, interpenetration and deceleration of the plumes cannot be modelled by standard hydrodynamic methods and a more advanced model has to be used. We employ the 1.5D multi-fluid code MULTIF which is designed for modelling of mixing, interpenetration and deceleration of several plasmas from different materials. The simulated velocity profiles of the $\mathrm{Al}$ ions reveal oscillations due to their deceleration and reflection. Those numerical results compare well with X-ray spectroscopic measurements performed at the PALS laser facility.
\end{abstract}

\section{INTRODUCTION}

Plasma-wall interactions (PWI) are important for inertial confinement and magnetic fusion. Here we contribute to the PWI studies by modelling the laser interaction with foils and subsequent interaction of two plasma plumes. The two foil target, see Fig. 1(c), consists of an upper Al foil of thickness $d_{u}=0.8 \mu \mathrm{m}$ and a lower $\mathrm{Mg}$ foil of thickness $d_{l}=2 \mu \mathrm{m}$. The foils are placed parallel at a distance $L=600 \mu \mathrm{m}$ (height $z=800 \mu \mathrm{m}$ and $200 \mu \mathrm{m}$ respectively in following figures). The Al foil is irradiated from above by a laser beam with wavelength $\lambda=0.438 \mathrm{~nm}$, energy $115 \mathrm{~J}$, full width at half maximum (FWHM) pulse length $300 \mathrm{ps}$, and focal spot radius $40 \mu \mathrm{m}$. The Mg foil is irradiated from below by a weaker laser (energy $6 \mathrm{~J}$, FWHM 300 ps, focal spot radius $25 \mu \mathrm{m}$ ).

\section{PRINCIPLES OF HYDRODYNAMICAL SIMULATIONS}

For the initial-stage modelling of laser interaction with a two-foil target we use the 2D hydrodynamics code PALE (Prague Arbitrary Lagrangian Eulerian) [1, 2] solving conservation laws for mass, momentum and energy for a single (non-interpenetrating), one temperature (i.e. electron and ion temperatures being the same) plasma. PALE simulations are run separately (with ideal-gas equation of state and laser absorption on a critical surface) for the counter-propagating $\mathrm{Al}$ and $\mathrm{Mg}$ plasma plumes (see Fig. 1(a), (b)) until they start to overlap, so that the $\mathrm{Al}$ and $\mathrm{Mg}$ plasmas should start to mix. At that point, from 2D maps of hydrodynamical quantities for each foil generated by PALE, 1D lineouts over $z$ taken at a fixed radius $r$ are extracted and combined, yielding a two-species description of colliding plasmas which is passed as an initial condition to the $1.5 \mathrm{D}$ multi-fluid hydrodynamics

This is an Open Access article distributed under the terms of the Creative Commons Attribution License 2.0, which permits unrestricted use, distribution, and reproduction in any medium, provided the original work is properly cited. 


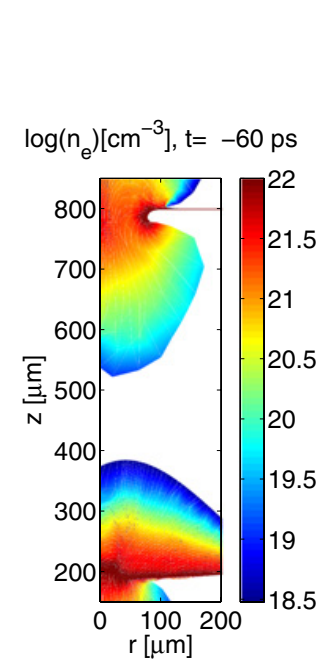

(a)

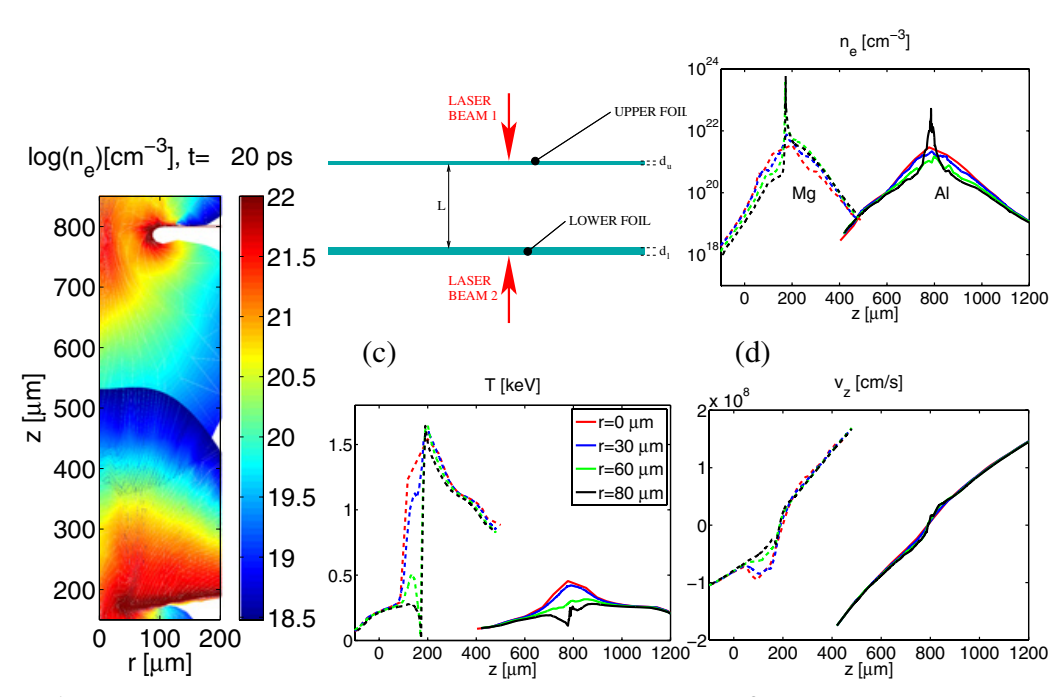

(e)

(f)

Figure 1. 2D PALE results: electron density $n_{e}$ at times $t=-60 \mathrm{ps}$ (a) and $t=+20 \mathrm{ps}$ (b); experimental setup (c); $1 \mathrm{D}$ cuts of PALE results, namely electron density $n_{e}(\mathrm{~d})$, temperature $T(\mathrm{e})$ and vertical velocity $v_{z}(\mathrm{f})$ at time $t=+20 \mathrm{ps}$ and fixed radii $r=0,30,60,80 \mu \mathrm{m}$. Solid lines in 1D cuts are for Al and dashed lines for Mg values.

code MULTIF [3]. All this is done for several values of $r$, and the corresponding MULTIF results are combined as described below to account for the full 2D plasma behaviour. MULTIF solves a set of mass, momentum and energy conservation equations for each material with an ideal-gas equation of state, with collisional drag terms exchanging momentum and energy between $\mathrm{Al}$ and $\mathrm{Mg}$ plasmas, as well as the neutralizing electron background. Electron thermal conduction uses a flux-limited Spitzer description. This model is derived from a Vlasov-Fokker-Planck formalism described in Ref. [4], in which a detailed discussion of the physics of interpenetrating plasmas can also be found.

\section{DISCUSSION OF SIMULATED DATA AND THEIR COMPARISON WITH EXPERIMENT}

PALE has been run till the time +20 ps (all times are related to the maximum of the laser pulse), i.e., time period after which the counter-propagating plasma overlap cannot be neglected. As an example of computed quantities we present in Fig. 1(a), (b) 2D distribution of electron density $n_{e}$ at times $t=$ $-60,+20$ ps. Two plumes expand from each foil, one of them upwards and the other one downwards. In Fig. 1(a), (b) we concentrate to the region between the foils, because we are interested in the interaction of $\mathrm{Al}$ plume propagating downwards from the upper $\mathrm{Al}$ foil with the $\mathrm{Mg}$ plume propagating upwards from the lower $\mathrm{Mg}$ foil. The $1 \mathrm{D}$ cuts of $n_{e}$, temperature $T$ and longitudal velocity $v_{z}$ at time $t=+20 \mathrm{ps}$ at fixed radii $r=0,30,60,80 \mu \mathrm{m}$ presented in Fig. 1 (d), (e), (f) served as initial conditions for the MULTIF runs. In the overlapping region of the $\mathrm{Al}$ and $\mathrm{Mg}$ plumes given by $z \in(400,500) \mu \mathrm{m}$ the corresponding $\mathrm{Al}$ and $\mathrm{Mg}$ densities are small, i.e., we can still neglect the collisional drag and assume that plumes interpenetrate without change in their velocities. Note that velocities in the overlapping region differ considerably.

These 1D cuts of PALE results were used as initial conditions for four runs (one at each radius) of MULTIF code simulating plumes interpenetration. The time evolution of plasma parameters (ion densities $n_{i}^{M g}, n_{i}^{A l}$, temperatures $T_{i}^{M g}, T_{i}^{A l}$ and longitudinal $z$ velocities $v_{i}^{M g}, v_{i}^{A l}$ along the $z$ axis) obtained for the MULTIF run on the $z$ axis, i.e. at $r=0 \mu \mathrm{m}$, is presented in Fig. 2 for both $\mathrm{Al}$ and $\mathrm{Mg}$ ions. In the beginning of the MULTIF simulation, the $\mathrm{Al}$ and $\mathrm{Mg}$ plasmas continue to interpenetrate 

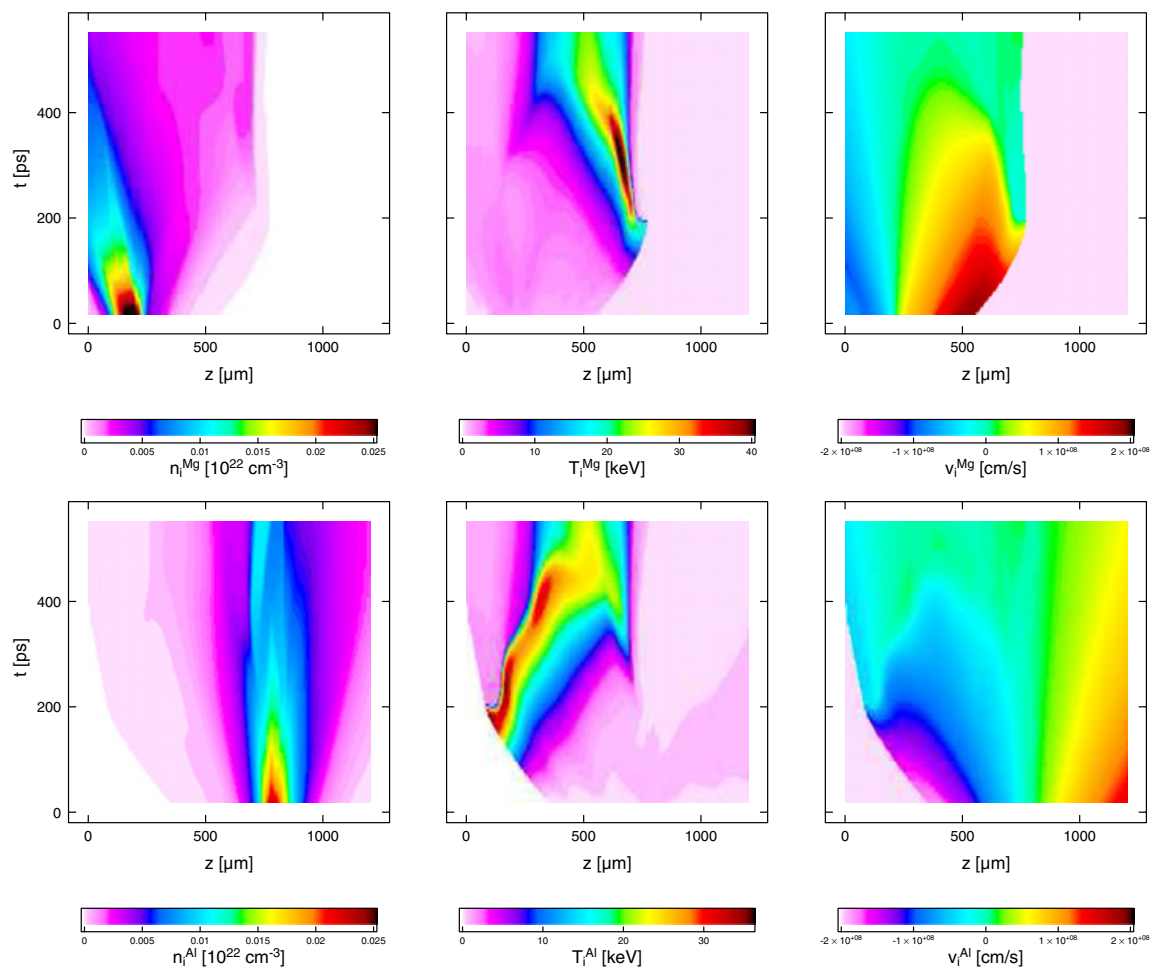

Figure 2. Time evolution of ion densities $n_{i}^{M g}, n_{i}^{A l}$ (left), ion temperatures $T_{i}^{M g}, T_{i}^{A l}$ (middle) and ion longitudinal $z$ velocities $v_{i}^{M g}, v_{i}^{A l}$ (right) for $\mathrm{Mg}$ (top) and $\mathrm{Al}$ (bottom) plasmas. $\mathrm{Al}$ foil is originally placed at $z=800 \mu \mathrm{m}$ and $\mathrm{Mg}$ foil at $z=200 \mu \mathrm{m}$.

without visible change in their velocity confirming that the collisional drag is still too small to influence their relative velocity. Looking at the left-hand border of the Al plasma we can notice that around time $t=80 \mathrm{ps}$ the $\mathrm{Al}$ ions temperature $T_{i}^{A l}$ starts to increase. The $\mathrm{Al}$ ions reach the region of higher $\mathrm{Mg}$ ions density, collisional drag starts to act and decelerate $\mathrm{Al}$ ions converting their kinetic energy into internal one, thus increasing $\mathrm{Al}$ ions temperature (here the $\mathrm{Mg}$ ions density is much higher than that of $\mathrm{Al}$, so $\mathrm{Mg}$ temperature changes only very little). Similar scenario appears a bit later around time $t=120 \mathrm{ps}$ on the right-hand border of $\mathrm{Mg}$ plasma (around $z=650 \mu \mathrm{m}$ at that time) where low density $\mathrm{Mg}$ ions reach higher density $\mathrm{Al}$ plasma and $\mathrm{Mg}$ ions are heated by collisional drag which decelerates them. The simulated thickness of the resulting high-temperature zones is a few tens of microns, which compares well with the ion mean free path calculated from slowing-down relaxation rates (taken from Ref. [5]) corresponding to the interaction of the $\mathrm{Al}$ ions with the counter-streaming Mg plasma.

Reconstructed spatially resolved emission spectrum of the Al Ly $\alpha$ group measured by a vertical dispersion Johann spectrometer is presented in Fig. 3(a) [6]. Spectra were recorded in such a way that Doppler shifts of spectral lines could be used to measure ion longitudinal $z$ velocity component orthogonal to the target. The optically thin Al Ly $\alpha$ J-satellite (marked in Fig. 3(a)) is practically not affected by the radiation transport, i.e., its observed wavelength shift can be used to measure the $\mathrm{Al}$ ions velocity. These velocities (with experimental error bars) derived from the shown spectra at different longitudinal $z$ positions are presented in Fig. 3(b) (dashed line). Most of the J-satellites are Dopplershifted to red thus resulting in negative $v_{i}^{A l}$ velocities (ions streaming to left-hand side), while close to the original position of the Mg foils at $z=200 \mu \mathrm{m}$ at several places the blue-shifts result in positive $v_{i}^{A l}$ velocities (ions flowing to the right). Let us recall that the spectra shown in Fig. 3(a), as well as 

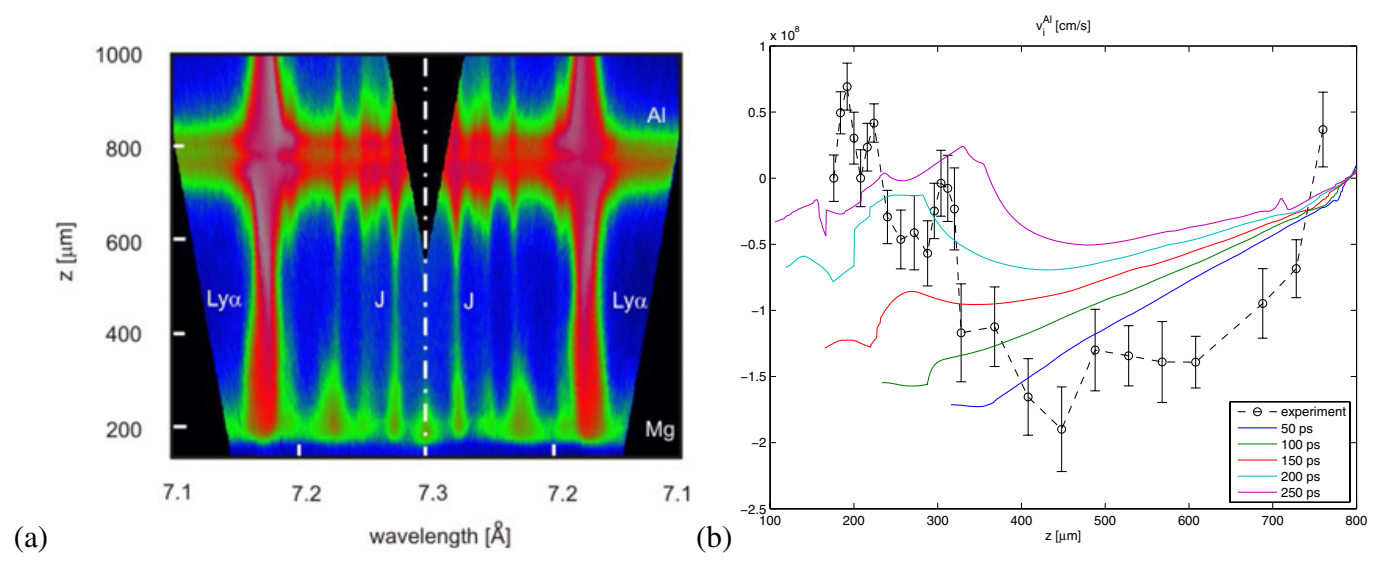

Figure 3. Spatially resolved spectrum of the Al Ly $\alpha$ spectral group recorded at the double-side laser-irradiated two-foil target (a). Experimentally observed effective Al ion velocities derived from Doppler shifts of the J-satellite and MULTIF velocity profiles at times 50,100,150,200, 250 ps (b). Initial foil positions: Al: $800 \mu \mathrm{m}, \mathrm{Mg}: 200 \mu \mathrm{m}$.

the reconstructed curve in Fig. 3(b), represent X-ray emission integrated over a long time (much longer than the duration of our simulations). The measured J-satellite positions (and shifts) then depend on the plasma parameters (density, temperature and ionization) evolving over time at each given $z$ position.

On the other hand, MULTIF results are instantaneous snapshots. In Fig. 3(b) are also displayed such MULTIF snapshots of the Al velocity profile at several times. These are averaged results from runs at $r=0,30,60,80 \mu \mathrm{m}$ radii, weighted by the density of $\mathrm{Al}$ ions. To get the effective simulation velocity results fully comparable with the experimental time-integrated values, one would need to post-process the MULTIF simulation results to recover the time-integrated profile of the J-satellite emission from the evolution of the plasma parameters. Indeed, at a given position $z$, the time-integrated velocity is expected to be mostly influenced by the plasma parameters recorded at the time when the J-satellite emission was the strongest. Although such a detailed post-processing of our simulation results is still a work in progress, we can already convince ourselves that the oscillations recorded in the time-integrated $\mathrm{Al}$ velocity profile in the vicinity of the $\mathrm{Mg}$ foil (originally at $z=200 \mu \mathrm{m}$ ) very likely result from an appropriate averaging of the individual profiles displayed in Fig. 3(b). We thus account for those oscillations as resulting from the detailed evolution of the process of slowingdown and stagnation of the Al fluid due to its collisional interaction with the counter-streaming Mg plasma.

\section{CONCLUSION}

Interpenetrating laser-produced plasmas were modelled by a combination of the 2D hydrodynamic code PALE with the 1.5 D multi-fluid code MULTIF. Simulated scenario of Al ions deceleration close to the secondary target explains the origin of the experimentally observed velocity oscillations. Successful simulations of ion velocity profiles close to the secondary targets contribute to plasma-wall interaction studies.

This research has been supported in part by the Czech Science Foundation Grant P205/10/ 0814, the CNRS PICS project No. 4343 and the Czech Ministry of Education project RVO 68407700. 


\section{References}

[1] R. Liska, J. Limpouch, M. Kuchařík, O. Renner, J. Phys.: Conf. Series 112, 022009 (2008).

[2] T. Kapin, M. Kuchařík, J. Limpouch, R. Liska, P. Váchal, Int. J. Num. Meth. Fluids 56, 1337 (2008).

[3] C. Chenais-Popovics, P. Renaudin, O. Rancu, F. Gilleron, J. Gauthier, O. Larroche, O. Peyrusse, M. Dirksmöller, P. Sondhauss, T. Missalla, I. Uschmann, E. Förster, O. Renner, E. Krouský, Phys. Plasmas 4, 190 (1997).

[4] O. Larroche, Phys. Fluids B 5, 2816 (1993).

[5] J. D. Huba, NRL plasma formulary, NRL/PU/6790-09-523, Washington (2009), p. 32.

[6] O. Renner, E. Krouský, R. Liska, M. Šmíd, O. Larroche, E. Dalimier, F. Rosmej , J. Phys.: Conf. Series 244, 022024 (2010). 